Received January 29, 2021; Accepted March 17, 2021;

Published online on March 19, 2021

DOI: 10.2116/analsci.21P047

Original Papers

\title{
Detection of Soluble Mercury in Cinnabar Using CV-Ag NPs SERS
}

\section{Probe}

Nan LI, *** Siqingaowa HAN, ${ }^{*}, * * \dagger$ Shuang LIN, ${ }^{*}$ Xuan-yu SHA, ${ }^{*}$ and Wuliji HASI*广

* National Key Laboratory of Science and Technology on Tunable Laser, Harbin Institute of Technology, Harbin 150080, China

** College of Art and Sciences, Northeast Agricultural University, Harbin 150030, China

*** Affiliated Hospital of Inner Mongolia University for Nationalities, Tongliao 028043, China

$\uparrow$ To whom correspondence should be addressed.

E-mail: hansiqin@126.com, hasiwuliji@126.com 


\begin{abstract}
In this paper, the uniform morphological Ag nanoparticles (Ag NPs) were prepared with ascorbic acid as the reducing agent and citrate as the stabilizer. The surface of Ag NPs modified by crystal violet $(\mathrm{CV})$ and potassium iodide $(\mathrm{KI})$ is used as the aggregation agent to obtain CV modified Ag NPs (CV-Ag NPs) probes for detecting mercury ions. The mercury ions can be reduced to mercury molecules by citrate, and then deposited them on the surface of Ag NPs, leading to the separation of CV molecules from the surface of Ag NPs. Therefore, the SERS signal intensity of CV decreases with the increase of $\mathrm{Hg}^{2+}$ concentration and the concentration of $\mathrm{Hg}^{2+}$ is in the range of $1 \times 10^{-11}$ to $1 \times 10^{-5} \mathrm{M}$. Taking the change of the characteristic peak intensity of $\mathrm{CV}$ at $913 \mathrm{~cm}^{-1}$ as a reference, the SERS spectrum intensity of CV has a linear relationship with the $\mathrm{Hg}^{2+}$ concentration. The equation is $y=-333.55^{*} x+1343.05$ with the linear correlation coefficient is $R^{2}=0.980$, and the recovery rate is between $84.20 \%$ to $105.60 \%$. Finally, the CV-Ag NPs probe was used to quickly detect the soluble mercury in cinnabar. Compared with the conventional large-scale instrument detection method, this simple and fast method, can be applied for the rapid detection of soluble mercury, and has a certain significance for the research of mineral medicine processing mechanism.
\end{abstract}

Keywords Surface-enhanced Raman spectroscopy; Ag nanoparticles; cinnabar; crystal violet; mercury ion 


\section{Introduction}

Traditional Chinese medicine (TCM) is a medical theory gradually formed by the people of our country in the course of thousands of years of development, following the laws of nature and rationally using natural resources. It is also an important component of our traditional culture. There are many kinds of TCM materials and their sources are wide. According to their sources, they can be divided into plants, minerals and animals. Cinnabar is a sulfide mineral medicine, mainly containing mercury sulfide (HgS), its content is not less than $96 \%$, and it also contains some soluble mercury, so it has certain toxic side effects. ${ }^{1}$ The cinnabar preparation method used in clinical practice is mostly the water processing method, which can effectively remove the soluble mercury in cinnabar. ${ }^{2}$ Some scholars also believe that mercury sulfide, the main component of cinnabar, is insoluble in water and difficult to be absorbed by the human body, so its medicinal effect comes from the soluble part. ${ }^{3}$ Therefore, the detection of cinnabar components, especially the study of its soluble mercury content, is of certain significance to ensure the safety of cinnabar drugs and to explore the processing mechanism. At present, lot of research effort was put in the exploration of dissolution of cinnabar, ${ }^{4,5}$ the analysis and testing of cinnabar mainly use the inductively coupled plasma-mass spectrometry (ICP-MS), ${ }^{6,7}$ Ultra-high liquid chromatography-inductively coupled plasma mass spectrometry. (UPLC-ICP/MS). ${ }^{8}$ The detection results of these methods are accurate and reliable, but generally require complicated sample extraction and separation processes. The detection time is long, unable to meet the on-site and fast detection requirements. Therefore, the study of a convenient, rapid and highly sensitive detection method has potential application prospects for the detection of soluble mercury in cinnabar.

Surface-Enhanced Raman Scattering (SERS) is a simple and efficient analysis technique. By using Au, Ag and other noble metal nanostructures as the SERS substrate, it can significantly enhance the Raman signal to detect low-concentration analytes. Even single molecules can be detected. ${ }^{9-11}$ When the SERS substrate interacts with 
electromagnetic waves, the local surface plasmons excited by the electromagnetic waves on the rough metal surface causes the electric field near the surface to amplify and produce hotspots, which increases the SERS signal intensity. ${ }^{12,}{ }^{13}$ With the development of SERS technology, some scholars have used the preparation of functionalized SERS substrates to detect heavy metal ions. For example, Yang's research group prepared inositol hexaphosphate $\left(\mathrm{IP}_{6}\right)$ as a stabilizer and surfacemodified crystal violet (CV) Au nanoparticles (Au NPs), by using trisodium citrate (TC) as a reducing agent to determine mercury ions in aqueous solutions. The limit of detection (LOD) of this method for mercury ions in aqueous solution was up to 0.5 pM. ${ }^{14}$ Wang's research group used Ag colloids as a reinforcing substrate to directly perform SERS detection on $\mathrm{Hg}^{2+}$ solution and found that the interaction between $\mathrm{Hg}^{2+}$ and Ag nanoparticles (Ag NPs) resulted in the formation of uneven mercury/Ag shells on the surface of Ag NPs. Affect the surface plasmons resonance (SPR) performance of Ag NPs. ${ }^{15}$ At present, SERS technology is mainly used to study mercury ions in rivers, lakes and seawater, ${ }^{16,17}$ and there have been no reports on the field of mineral medicine containing soluble mercury. Therefore, the study of a convenient, rapid and highly sensitive detection method has potential application prospects for the detection of soluble mercury in cinnabar.

In this study, CV-Ag NPs probes were obtained by preparing Ag nanoparticles with uniform morphology and modifying $\mathrm{CV}$ molecules, using the reduction effect of citrate on mercury ions, ${ }^{18}$ causing mercury molecules to be adsorbed on the surface of Ag NPs, replacing CV The SERS signal of molecule-induced CV gradually weakened with the increase of mercury ion concentration. By testing the signal enhancement effects of different aggregation agents, KI was selected as the aggregation agent, and the characteristic peak of $\mathrm{CV}$ at $913 \mathrm{~cm}^{-1}$ was used as a reference to study the relationship between mercury ion concentration and the change of peak intensity, and finally, CVAg NPs have used the probe quickly detects soluble mercury in cinnabar. Compared with conventional detection methods based on large-scale instruments, this method is simple and rapid and is suitable for the rapid detection of soluble mercury in mineral medicines. 


\section{Experimental}

\section{Reagents and Instrumental}

Silver nitrate $\left(\mathrm{AgNO}_{3}\right)$, trisodium citrate $\left(\mathrm{C}_{6} \mathrm{H}_{5} \mathrm{Na}_{3} \mathrm{O}_{7}\right)$ were obtained from Sinopharm Chemical Reagent Co., Ltd. Ascorbic acid (AA) was obtained from Acros Organics Co., Ltd. Crystal violet (CV) obtained from Tianjin Guangfu Fine Chemical Research Institute, Potassium iodide (KI), Sodium chloride $(\mathrm{NaCl})$ were obtained from Xilong Scientific Co., Ltd. Sodium Bromide (NaBr) was obtained from TCI Shanghai Chemical Industry Development Co., Ltd. Mercury chloride $\left(\mathrm{HgCl}_{2}\right)$ was purchased from Jiangyan Huanqiu Reagent Factory. Cinnabar 1 was purchased from the Mongolian Medicine Preparation Room of the Affiliated Hospital of Inner Mongolia University for Nationalities, cinnabar 2 was purchased from the Harbin Sankeshu Chinese Medicinal Material Market. Milli Q water $(>18.0 \mathrm{M} \Omega \mathrm{cm})$ was purified with a Sartorius Arium 611 UV ultrapure water system. All chemicals were used without further purification.

The SERS spectrometer is a BWS415-785H (B\&W Tek, INC.) portable Raman spectrometer, the excitation wavelength was $785 \mathrm{~nm}$. The spectrum measurement range is 68 to $2700 \mathrm{~cm}^{-1}$, and the spectral resolution is better than $3 \mathrm{~cm}^{-1}$. Spectral data collection and related processing use the software Bwram1.01.20 attached to the spectrometer. The ultraviolet-visible absorption spectrophotometer is GENESYS 50 (Thermo Fisher Scientific) ultraviolet-visible spectrophotometer. The morphological characterization instrument is SU8010 (Hitachi) cold field emission scanning electron microscope (SEM), and the accelerating voltage range is 0.1 to $30 \mathrm{kV}$.

\section{Preparation of Ag nanoparticles}

Ag NPs are prepared by Qin's method. ${ }^{19}$ The specific experimental steps are as follows: Take $8.0 \mathrm{~mL}$ of an aqueous solution containing AA $\left(6.0 \times 10^{-4} \mathrm{M}\right)$ and trisodium citrate $\left(3.0 \times 10^{-3} \mathrm{M}\right)$. Then, $0.08 \mathrm{~mL}$ of silver nitrate $(0.1 \mathrm{M})$ solution was added under 
stirring at $900 \mathrm{rpm}$ in a $30^{\circ} \mathrm{C}$ water bath, and it was observed that the reaction solution changed from colorless to yellow and then to brown. After the completion of the reaction, it was matured in a water bath of $100{ }^{\circ} \mathrm{C}$ for 2 hours to finally obtain $\mathrm{Ag}$ colloid.

\section{Sample preparation and SERS detection}

The preparation process of the CV-Ag NPs probe is shown in Figure 1, mixing $6 \mathrm{ml}$ of Ag colloids with $6 \mathrm{ml}$ of $\mathrm{CV}$ solution to obtain the $\mathrm{CV}-\mathrm{Ag}$ NPs probe, where $\mathrm{CV}$ as the probe molecule concentration is $1 \times 10^{-6} \mathrm{M}$. The $\mathrm{Hg}^{2+}$ solution is prepared by

dissolving $\mathrm{HgCl}_{2}$ in ultrapure water. First, prepare a $1 \times 10^{-2} \mathrm{M} \mathrm{Hg}^{2+}$ solution, and then dilute sequentially to obtain the concentrations of $1 \times 10^{-3}, 1 \times 10^{-4}, 1 \times 10^{-5}, 1 \times 10^{-6}, 1 \times 10^{-}$ ${ }^{7}, 1 \times 10^{-8}, 1 \times 10^{-9}, 1 \times 10^{-10}$ and $1 \times 10^{-11} \mathrm{M}$ gradient concentration $\mathrm{Hg}^{2+}$ Solution.

The cinnabar sample was processed by the water processing method (called Shui-Fei in Chinese). ${ }^{2}$ The specific steps are as follows: first, $1 \mathrm{~g}$ of cinnabar sample was weighed and a proper amount of deionized water was added and grinded to a paste. Then a lot of water repeatedly grind ( $50 \mathrm{ml}$ total water) and after the solution becomes a red suspension, the supernatant after precipitation was taken for detection. (Fig. S1, Supporting Information).

When using the CV-Ag NPs probe for detection, take $20 \mathrm{~mL}$ mercury ion solution of different concentrations and $20 \mathrm{~mL} \mathrm{CV}$-Ag NPs probe solution to mix thoroughly, and then use Raman spectrometer to detect. Unless otherwise noted, the detection conditions of the spectrometer are laser power $30 \mathrm{~mW}$, integration time $3 \mathrm{~s}$. Spectral data collection and related processing use the software Bwram1.01.20 that comes with the spectrometer. Figure 1 is a schematic diagram of the detect process.

\section{Results and Discussion}

\section{Characterization of the Ag NPs}

Compared with the classic method of preparing Ag colloids by Lee and Meisel, the Ag colloids prepared by using ascorbic acid as the reducing agent has a higher yield of 
Ag NPs and no structures such as nanowires. ${ }^{[15]}$ A random selection method was used to select 100 nanoparticles from the Ag colloids scanning electron microscope image and the particle size distribution of the Ag colloids was obtained through statistical analysis. As shown in Figure 2(a), the diameter of the Ag NPs is 45.7 $\pm 7.1 \mathrm{~nm}$. Through the scanning electron micrograph of the Ag colloids (Figure 2(a) inset), it can be seen that the Ag NPs are mostly spherical and uniform in size. The ultraviolet absorption spectrum of Ag colloids is shown in Figure 2(b). The Ag colloids has a visible absorption peak at $400 \mathrm{~nm}$, which can be attributed to the plasma single-mode vibration of Ag NPs. ${ }^{20}$

\section{Surface-enhanced Raman spectroscopy of probe molecules}

CV molecules have high SERS activity, they are positively charged and can be electrostatically adsorbed on the surface of Ag NPs coated with citrate, so they are widely used as probe molecules in the field of SERS. ${ }^{21}$ First we took an appropriate amount of CV solution with a concentration of $1 \times 10^{-2} \mathrm{M}$ on the aluminum foil tape and use a portable Raman spectrometer to perform routine Raman detection. And then the $\mathrm{CV}$ solution with a concentration of $1 \times 10^{-7} \mathrm{M}$ was mixed with $\mathrm{Ag}$ colloids. An appropriate amount of the solution is tested on the aluminum foil tape. The SERS detection results and the molecular structure of $\mathrm{CV}$ are shown in Figure 3. The SERS spectrum of CV has obvious characteristic peaks at 725, 805, 913 and $1173 \mathrm{~cm}^{-1}$. The characteristic peak at $725 \mathrm{~cm}^{-1}$ corresponds to out-of-plane vibrations of ring $\mathrm{C}-\mathrm{H}$, the characteristic peak at $805 \mathrm{~cm}^{-1}$ corresponds to $\mathrm{CH}$ out-of-plane swing, the characteristic peak at $913 \mathrm{~cm}^{-1}$ corresponds to ring skeletal vibration of radical orientation, and the characteristic peak at $1173 \mathrm{~cm}^{-1}$ corresponds to in-plane vibrations of ring C-H. ${ }^{22}$ Based on the above-mentioned characteristic peaks of $\mathrm{CV}$ molecules, combined with the conventional Raman spectrum of CV solution, the analytical enhancement factor of the Ag colloids was calculated. The calculation results are shown in Table 1. It can be seen that compared with ordinary Raman spectroscopy, the enhancement factor of the SERS spectrum is in the range of $0.71 \times 10^{6}$ to $1.76 \times 10^{6}$, and the characteristic peak at $913 \mathrm{~cm}^{-}$

${ }^{1}$ has the best enhancement effect. In the follow-up work, the characteristic peak at 913 
$\mathrm{cm}^{-1}$ is used for corresponding research.

\section{The effect of different aggregation agents}

Studies have shown that the local electric field intensity generated by multiple noble metal nanoparticles in close contact is much greater than the local electric field intensity generated by a single noble metal nanoparticle. Therefore, when using noble metal colloids substrates for SERS detection, aggregation agents are often used to make precious metal nanoparticles aggregated, thus producing hotspots. ${ }^{23}$ By adding halide ions to the Ag colloids, the negatively charged citrate on the surface of the nanoparticles can be replaced, breaking the potential balance of the colloids system, so that the nanoparticles in the Ag colloids can aggregate to produce hotspots. ${ }^{24}$ In this study, $1 \mathrm{M}$ $\mathrm{NaBr}, \mathrm{KI}$ and $\mathrm{NaCl}$ inorganic salt solutions were selected as aggregation agents for comparison, and the same amount was added dropwise to the mixed solution of $1 \times 10^{-6}$ M CV-Ag NPs probe. The solution $15 \mu \mathrm{L}$ was placed on the aluminum foil tape for SERS detection and the result is shown in Figure 4(a). It can be seen from the figure that after adding the aggregation agent, the enhancement effect of the Ag colloids has been significantly improved. To intuitively compare the signal enhancement effects of the above three aggregation agents, the characteristic peak intensities at 725, 805, 913 and $1173 \mathrm{~cm}^{-1}$ of the $\mathrm{CV}$ solution with different aggregation agents are compared, as shown in Figure 4(b). It can be seen that at the same concentration $(1 \mathrm{M})$, the KI solution of the above three aggregation agents has the best enhancement effect, which may be caused by the different adsorption characteristics of $\mathrm{I}^{-}, \mathrm{Cl}^{-}$and $\mathrm{Br}^{-}$ions. The inorganic salts can play a good role in enhancing. ${ }^{25}$

\section{The sensitivity of mercury ion detection}

To test the detection sensitivity of the CV-Ag NPs probe to $\mathrm{Hg}^{2+}, \mathrm{Hg}^{2+}$ solutions of $1 \times 10^{-11}$ to $1 \times 10^{-3} \mathrm{M}$ were added to the $\mathrm{CV}-\mathrm{Ag} \mathrm{NPs}$ solution for detection. Each concentration is tested 5 times and averaged to calculate the corresponding error bar. The detection results of CV-Ag NPs solutions containing different concentrations of $\mathrm{Hg}^{2+}$ are shown in Figure 5(a). It can be seen that as the concentration of mercury ions 
increases, the SERS signal of CV gradually weakens. Using the characteristic peak intensity of the $\mathrm{CV}$ at $913 \mathrm{~cm}^{-1}$ as a reference, the relationship between the characteristic peak intensity and the concentration change is obtained (Figure 5(b)). It can be seen from Figure 5(b), within the concentration range of $1 \times 10^{-11}$ to $1 \times 10^{-5} \mathrm{M}$, the SERS spectral intensity of CV presented a linear relationship with the concentration of $\mathrm{Hg}^{2+}$. Through fitting, $y=-333.55^{*} x+1343.05$, linear correlation coefficient $R^{2}=0.980$. Also, to test the limit of detection (LOD) of the method, the characteristic peak intensity of the blank CV-Ag NPs probe at $913 \mathrm{~cm}^{-1}$ minus 3 times the signal-to-noise ratio is used to calculate the LOD as $2.28 \times 10^{-10} \mathrm{M}^{26}$

To verify the reliability and accuracy of the detection method, the spiked mercury ion solutions with concentrations of $0.5 \times 10^{-10}$ and $3.0 \times 10^{-8} \mathrm{M}$ were configured, and the CV-Ag NPs probe was used for SERS detection, and the fitting curve was used to determine. The recovery rate and relative standard deviation of this method are calculated. The calculation results are shown in Table 2 . The recovery rate of mercury ions in the solution detected by the CV-Ag NPs probe is between $84.32 \%$ to $105.6 \%$, and the RSD is less than $11 \%$. It can be seen that this method has high sensitivity and recovery rate, which ensures the efficiency and accuracy of the detection method.

\section{The influence of other heavy metal cations on CV-Ag NPs probe}

In addition to mercury, soluble heavy metals in mineral medicines such as cinnabar also include cadmium $(\mathrm{Cd})$, lead $(\mathrm{Pb})$, arsenic $(\mathrm{As})$, iron $(\mathrm{Fe})$ and antimony $(\mathrm{Sb}){ }^{27,} 28$ Therefore, to test the influence of other heavy metal cations on the CV-Ag NPs probe, different heavy metal cations were added to the CV-Ag NPs probe solution under the same conditions to test the selectivity of the detection method. The selected heavy metal cations are: $\mathrm{Cd}^{2+}, \mathrm{Pb}^{2+}, \mathrm{As}^{3+}, \mathrm{As}^{5+}, \mathrm{Fe}^{3+}$ and $\mathrm{Sb}^{3+}$, and the concentrations are all $1 \times 10^{-6}$ M. Figure 6 shows the difference of the characteristic peak intensity of CV-Ag NPs probe at $913 \mathrm{~cm}^{-1}$ before and after adding different metal cations. It can be seen that mercury ion $\left(1 \times 10^{-7} \mathrm{M}\right)$ significantly reduces the characteristic of $\mathrm{CV}$ at $913 \mathrm{~cm}^{-1}$ peak intensity, and other heavy metal cations did not cause the Raman intensity change of the probe molecule, which can indicate that the CV-Ag NPs probe has good selectivity 
for mercury ions.

\section{Detection of soluble mercury in cinnabar}

In the past, SERS technology has been used to detect mercury ions in the environment (Table S1, Supporting Information). In order to test the soluble mercury content in the TCM, the study used the CV-Ag NPs probe to detect the cinnabar samples. The cinnabar sample was pretreated and its supernatant was used for detection with SERS probe. Then the concentration of mercury ions in the supernatant was calculated based on the fitted curve and the intensity of the Raman characteristic peak with $\mathrm{CV}$ at $913 \mathrm{~cm}^{-1}$. The calculation results are shown in Table 3 . The concentrations of mercury ions in the supernatant are $6.220 \times 10^{-7}$ and $5.224 \times 10^{-6} \mathrm{M}$, and after conversion, the soluble mercury content in cinnabar 1 and cinnabar 2 is 0.624 and $5.140 \mu \mathrm{g} / \mathrm{g}$, respectively.

Compared with other large-scale instruments for detecting mercury in TCM (Table 4), ${ }^{29,30}$ the detection limit and accuracy of detection of soluble mercury in mineral medicines using SERS technology are similar. However, the SERS detection method has simple pretreatment, a simple and fast detection process, and has great practical application value for detecting soluble mercury in mineral medicine.

\section{Conclusion}

In this paper, high-yield Ag NPs were obtained by adding a ripening step in the preparation of Ag colloids, and CV molecules were modified on the surface of Ag NPs to prepare $\mathrm{CV}-\mathrm{Ag}$ NPs probes to detect mercury ions. By using KI as an aggregation agent, the SERS signal enhancement factor of the CV-Ag NPs probe can be as high as $1.76 \times 10^{6}$. In the concentration range of $1 \times 10^{-11}$ to $1 \times 10^{-5} \mathrm{M}$, the $\mathrm{Hg}^{2+}$ concentration is comparable to that of the CV-Ag NPs probe. The SERS spectral intensity fitting equation is $y=-333.55^{*} x+1343.05$, and the linear correlation coefficient $R^{2}=0.980$. Finally, the CV-Ag NPs probe was used to determine the soluble mercury in cinnabar. The test results show that the soluble mercury content in cinnabar from different sources 
is different. Compared with conventional large-scale instrument detection methods, this method is simple and rapid and has a certain significance for the rapid detection of soluble mercury in mineral medicines.

\section{Acknowledgements}

This work was supported by the National Natural Science Foundation of China (Grant No. 31871873) and the Inner Mongolia Autonomous Region Natural Science Foundation of China (Grant No. 2018LH08055).

\section{References}

1. X. Zhou, L. Wang, X. Sun, X. Yang, C. Chen, Q. Wang, and X. Yang, J. Ethnopharmacol., 2011, 135, 110 .

2. S. Cao, J. Xia, L. Li, H. Chen, X. Yang, and S. Ji, Lishizhen Medicine and Materia Medica Research, 2016, 27, 1110.

3. X. Zhou, K. Zeng, Q. Wang, X. Yang, and K. Wang, J. Ethnopharmacol., 2010, 131, 196.

4. P. Jiang, Y. Li, G. Liu, G. Yang, L. Lagos, Y. Yin, B. Gu, G. Jiang, and Y. Cai, J. Hazard. Mater, 2016, $317,466$.

5. J. S. Waples, K. L. Nagy, G. R. Aiken, and J. N. Ryan, Geochim. Cosmochim. Acta, 2005, 69, 1575.

6. R. J. Huang, Z. X. Zhuang, Y. Tai, R. F. Huang, X. R. Wang, and F. S. C. Lee, Talanta, 2006, 68, 728.

7. Q. T. Jiang, L. Zeng, J. Ma, L. N. Peng, W. L. Li, Y. Ding, and J. Li, Chinese Journal of Analytical Chemistry, 2016, 44, 979.

8. Q. Wu, X. He, S. J. Zhou, F. G. Shi, and Y. F. Lu, Toxicology in Vitro, 2020, 63, 7.

9. J. Langer, D. Jimenez De Aberasturi, J. Aizpurua, R. A. Alvarez-Puebla, B. Auguie, J. J. Baumberg, G.

C. Bazan, S. E. J. Bell, A. Boisen, A. G. Brolo, J. Choo, D. Cialla-May, V. Deckert, L. Fabris, K. Faulds, F. J. Garcia De Abajo, R. Goodacre, D. Graham, A. J. Haes, C. L. Haynes, C. Huck, T. Itoh, M. Kall, J. Kneipp, N. A. Kotov, H. Kuang, E. C. Le Ru, H. K. Lee, J. F. Li, X. Y. Ling, S. A. Maier, T. Mayerhofer, M. Moskovits, K. Murakoshi, J. M. Nam, S. Nie, Y. Ozaki, I. Pastoriza-Santos, J. PerezJuste, J. Popp, A. Pucci, S. Reich, B. Ren, G. C. Schatz, T. Shegai, S. Schlucker, L. L. Tay, K. G. Thomas, Z. Q. Tian, R. P. Van Duyne, T. Vo-Dinh, Y. Wang, K. A. Willets, C. Xu, H. Xu, Y. Xu, Y. S. Yamamoto, B. Zhao, and L. M. Liz-Marzan, ACS Nano, 2020, 14, 28.

10. A. B. Zrimsek, N. Chiang, M. Mattei, S. Zaleski, M. O. Mcanally, C. T. Chapman, A. I. Henry, G. C. Schatz, and R. P. Van Duyne, Chem. Rev., 2017, 117, 7583.

11. N. Li, S. Han, C. Zhang, S. Lin, X. Y. Sha, and W. Hasi, Anal. Sci., 2020, 36, 935.

12. S. Lin, X. Lin, S. Han, H. Zhao, W. Hasi, and L. Wang, Nanotechnology, 2019, 30, 215601.

13. X. Lin, G. Fang, Y. Liu, Y. He, L. Wang, and B. Dong, Journal of Physical Chemistry Letters, 2020, $11,3573$.

14. S. Y. Fu, X. Y. Guo, H. Wang, T. X. Yang, Y. Wen, and H. F. Yang, Sensors and Actuators B-Chemical, 
2014, 199, 108.

15. W. Ren, C. Zhu, and E. Wang, Nanoscale, 2012, 4, 5902.

16. Y. Qi, J. Zhao, G.-J. Weng, J.-J. Li, X. Li, J. Zhu, and J.-W. Zhao, Journal of Materials Chemistry C, 2018, $6,12283$.

17. K. Li, A. Liang, C. Jiang, F. Li, Q. Liu, and Z. Jiang, Talanta, 2012, 99, 890.

18. I. Ojea-Jimenez, X. Lopez, J. Arbiol, and V. Puntes, Acs Nano, 2012, 6, 2253.

19. Y. Q. Qin, X. H. Ji, J. Jing, H. Liu, H. L. Wu, and W. S. Yang, Colloids and Surfaces aPhysicochemical and Engineering Aspects, 2010, 372, 172.

20. P. Pinkhasova, H. Chen, M. W. G. M. Verhoeven, S. Sukhishvili, and H. Du, Rsc Advances, 2013, 3, 17954.

21. S. Lin, X. Lin, S. Q. G. W. Han, L. He, H. Y. Zhao, J. Zhang, W. L. J. Hasi, and L. Wang, J. Alloys Compd., 2019, 805, 318.

22. L. He, N.-J. Kim, H. Li, Z. Hu, and M. Lin, J. Agric. Food. Chem., 2008, 56, 9843.

23. S. Q. Han, X. Chen, C. Zhang, H. Zhao, S. Lin, Y. Zhang, and W. L. Hasi, Anal. Sci., 2019, 35, 1209.

24. C. Zhang, S. Q. Han, H. Zhao, S. Lin, and W. L. Hasi, Anal. Sci., 2018, 34, 1249.

25. L. Xie, J. Lu, T. Liu, G. Chen, G. Liu, B. Ren, and Z. Tian, J Phys Chem Lett, 2020, 11, 1022.

26. C. Zhu, G. Meng, P. Zheng, Q. Huang, Z. Li, X. Hu, X. Wang, Z. Huang, F. Li, and N. Wu, Adv. Mater, 2016, 28, 4871.

27. T. Kulikova, E. Hiller, L. Jurkovic, L. Filova, P. Sottnik, and P. Lacina, Environ. Monit. Assess., 2019, $191,263$.

28. R. L. Frost, S. Bahfenne, and E. C. Keeffe, Journal of Raman Spectroscopy, 2010, 41, 1779.

29. X. Yuan, K. Li, Y. Zhang, Y. Miao, Y. Xiang, Y. Sha, M. Zhang, and K. Huang, Microchem. J., 2020, $155,1$.

30. L. F. Shi, D. F. Xue, H. G. Xu, H. Liu, and W. F. Teng, Spectroscopy and Spectral Analysis, 2007, 27, 1036. 
Table 1. Analysis Enhanced factor (AEF) for different SERS peaks. ${ }^{\text {a }}$

\begin{tabular}{cccccc}
\hline $\begin{array}{c}\text { Raman Peak } \\
\left(\mathrm{cm}^{-1}\right)\end{array}$ & ISERS & $\begin{array}{c}K_{\text {SERS }} \\
(\mathrm{L} / \mathrm{mol})\end{array}$ & $I_{N R S}$ & $\begin{array}{c}K_{N R S} \\
(\mathrm{~L} / \mathrm{mol})\end{array}$ & AEF \\
\hline 725 & 4893 & $4.893 \times 10^{10}$ & 327 & $3.27 \times 10^{4}$ & $1.50 \times 10^{6}$ \\
805 & 2997 & $2.997 \times 10^{10}$ & 423 & $4.23 \times 10^{4}$ & $0.71 \times 10^{6}$ \\
913 & 4126 & $4.126 \times 10^{10}$ & 235 & $2.35 \times 10^{4}$ & $1.76 \times 10^{6}$ \\
1173 & 4670 & $4.670 \times 10^{10}$ & 390 & $3.90 \times 10^{4}$ & $1.20 \times 10^{6}$ \\
\hline
\end{tabular}

${ }^{\mathrm{a}}$ The $\mathrm{C}_{0}$ and $\mathrm{C}_{1}$ are the $\mathrm{CV}$ concentration of the normal Raman systems and the SERS system with $1.0 \times 10^{-2} \mathrm{M}$ and $1.0 \times 10^{-7} \mathrm{M}$ respectively. $K_{\text {SERS }}=I_{\text {SERS }} / \mathrm{C}_{1}, K_{N R S}=I_{N R S} / \mathrm{C}_{0}, \mathrm{AEF}=K_{\text {SERS }} / K_{N R S}$.

Table 2. Results for the determination of $\mathrm{Hg}^{2+}$ in samples

\begin{tabular}{ccccc}
\hline $\begin{array}{c}\text { Spiked } \\
\text { Concentration, M }\end{array}$ & Number & $\begin{array}{c}\text { Calculated } \\
\text { Concentration, } \\
\text { M }\end{array}$ & $\begin{array}{c}\text { Recovery, } \\
\%\end{array}$ & $\begin{array}{c}\text { RSD, } \\
\%\end{array}$ \\
\hline $5.0 \times 10^{-11}$ & 1 & $4.571 \times 10^{-11}$ & 91.42 & \\
& 2 & $4.735 \times 10^{-11}$ & 94.70 & 5.89 \\
& 3 & $4.216 \times 10^{-11}$ & 84.32 & \\
$3.0 \times 10^{-8}$ & 1 & $4.507 \times 10^{-11}$ & 90.15 & \\
& 2 & $2.603 \times 10^{-8}$ & 86.77 & \\
& 3 & $2.681 \times 10^{-8}$ & 89.37 & 10.88 \\
& Mean & $3.168 \times 10^{-8}$ & 105.60 & \\
\hline
\end{tabular}

Table 3. Results for the determination of $\mathrm{Hg}^{2+}$ in cinnabar

\begin{tabular}{lcc}
\hline Sample & Number & Calculated Concentration, M \\
\hline \multirow{2}{*}{ Cinnabar 1 } & 1 & $6.578 \times 10^{-8}$ \\
& 2 & $5.937 \times 10^{-8}$ \\
& 3 & $6.146 \times 10^{-8}$ \\
Mean & $6.220 \times 10^{-8}$ \\
& 1 & $5.861 \times 10^{-7}$ \\
& 2 & $5.159 \times 10^{-7}$ \\
& 3 & $4.352 \times 10^{-7}$ \\
& Mean & $5.124 \times 10^{-7}$ \\
\hline
\end{tabular}


Table 4. The comparison of methods for the detection of Hg in TCM.

\begin{tabular}{|c|c|c|c|c|}
\hline Method & $\begin{array}{l}\text { Limit of } \\
\text { detection }\end{array}$ & $\begin{array}{l}\text { Required } \\
\text { time for pre- } \\
\text { treatment }\end{array}$ & $\begin{array}{l}\text { Whether } \\
\text { require } \\
\text { large-scale } \\
\text { instruments }\end{array}$ & Reference \\
\hline $\begin{array}{l}\text { Low PowerPoint } \\
\text { discharge atomic } \\
\text { emission spectrometry } \\
\text { (PD-AES) }\end{array}$ & $0.15 \mathrm{ng} \mathrm{mL}^{-1}$ & $30 \sim 60 \mathrm{~min}$ & Yes & {$[26]$} \\
\hline $\begin{array}{c}\text { Inductively coupled } \\
\text { plasma mass } \\
\text { spectrometry (ICP-MS) }\end{array}$ & $0.044 \mathrm{ng} \mathrm{mL}^{-1}$ & 30 90 min & Yes & [27] \\
\hline SERS & $\begin{array}{l}0.045 \mathrm{ng} \mathrm{mL}^{-1} \\
\left(2.28 \times 10^{-10} \mathrm{M}\right)\end{array}$ & $10 \mathrm{~min}$ & No & This work \\
\hline
\end{tabular}




\section{Figure Captions}

Fig. 1 Schematic diagram of the CV-Ag NPs SERS probe proposed for mercury ion detection.

Fig. 2 (a) Particle size distribution of Ag NPs. Inset: SEM of Ag NPs, (b) UV-visible absorption spectra of Ag colloid. Inset: pictures of Ag colloid.

Fig. 3 Conventional Raman spectrum $\left(1 \times 10^{-2} \mathrm{M}\right)$ and SERS spectrum $\left(1 \times 10^{-7} \mathrm{M}\right)$ of $\mathrm{CV}$ solution, laser power 30 $\mathrm{mW}$, integration time $3 \mathrm{~s}$. Inset: molecular structure of CV.

Fig. 4 (a) SERS spectrum of CV solution $\left(1 \times 10^{-6} \mathrm{M}\right)$ under the action of different aggregating agents, laser power $30 \mathrm{~mW}$, integration time $3 \mathrm{~s}$, (b) Comparison of the characteristic peak intensities of CV solution $\left(1 \times 10^{-6} \mathrm{M}\right)$ at $725,805,913$ and $1173 \mathrm{~cm}^{-1}$ under the action of different aggregating agents.

Fig. 5 (a) Using CV-AgNPs probe to detect SERS spectra of $\mathrm{Hg}^{2+}$ solutions with different concentrations, laser power $30 \mathrm{~mW}$, integration time $3 \mathrm{~s}$, (b) $\mathrm{CV}$ characteristic peak $\left(913 \mathrm{~cm}^{-1}\right)$ intensity fitted with $\mathrm{Hg}^{2+}$ concentration curve.

Fig. 6 Use CV-Ag NPs probe to detect different heavy metal cations, $\mathrm{R}_{0}$ and $\mathrm{R}$ are the characteristic peak intensity of CV-Ag NPs probe at $913 \mathrm{~cm}^{-1}$ before and after adding heavy metal cations, laser power $30 \mathrm{~mW}$, integration time $3 \mathrm{~s}, \mathrm{CHg}^{2+}: 1 \times 10^{-7} \mathrm{M}, \mathrm{C}_{\text {other cations }}: 1 \times 10^{-6} \mathrm{M}$ 


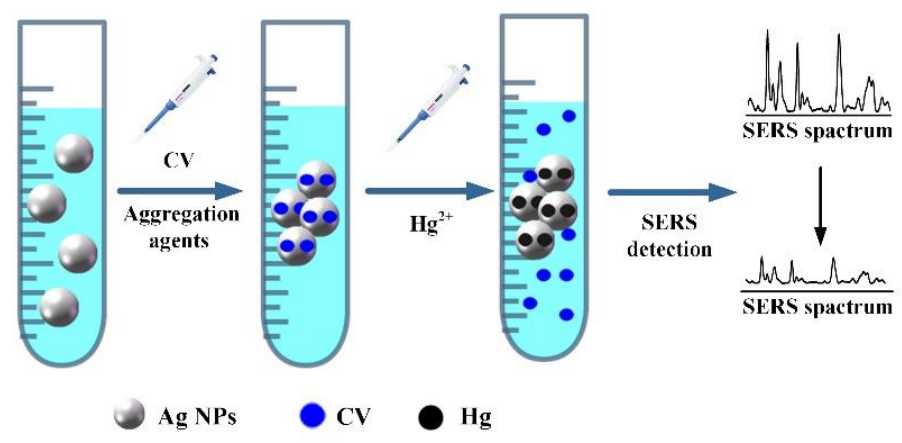

Fig. 1 Schematic diagram of the CV-Ag NPs SERS probe proposed for mercury ion detection.
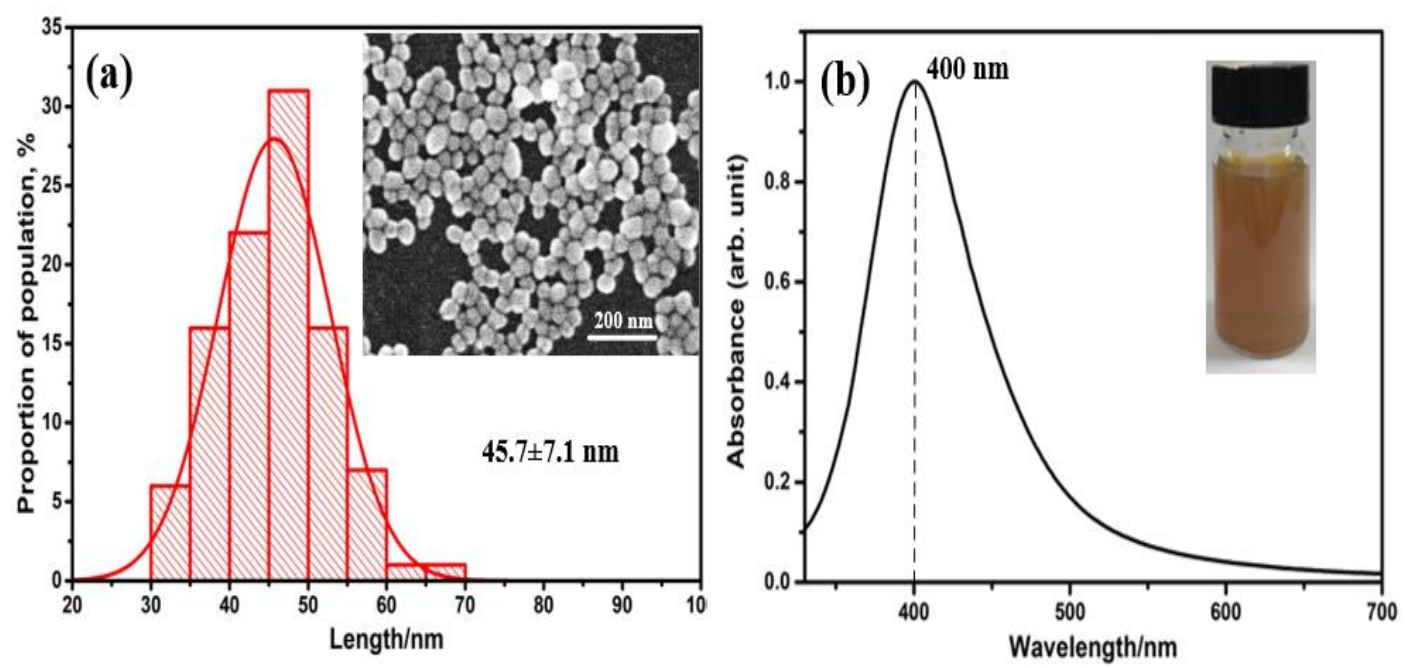

Fig. 2 (a) Particle size distribution of Ag NPs. Inset: SEM of Ag NPs, (b) UV-visible absorption spectra of Ag colloid. Inset: pictures of Ag colloid.

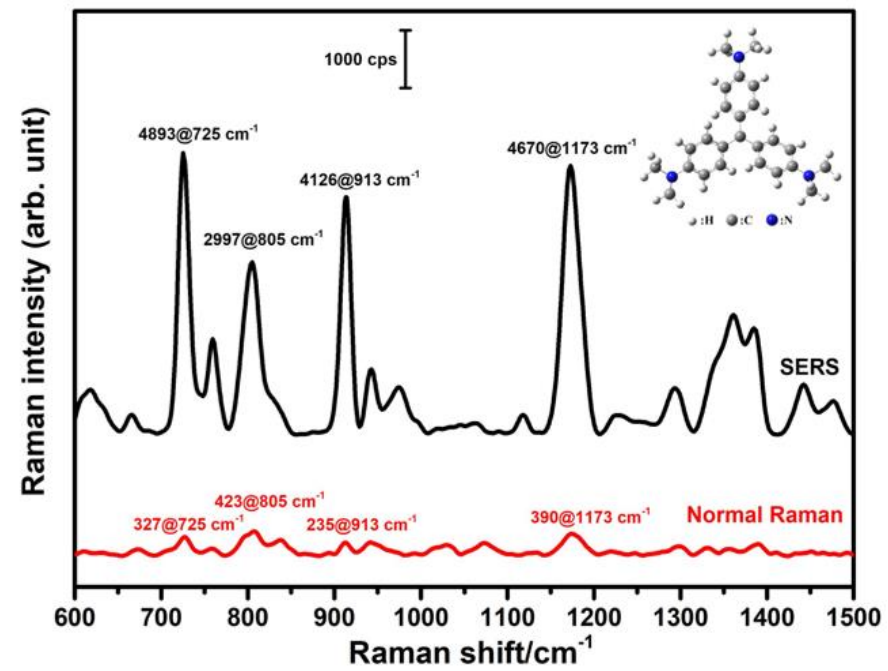

Fig. 3 Conventional Raman spectrum $\left(1 \times 10^{-2} \mathrm{M}\right)$ and SERS spectrum $\left(1 \times 10^{-7} \mathrm{M}\right)$ of CV solution, laser power 30 $\mathrm{mW}$, integration time $3 \mathrm{~s}$. Inset: molecular structure of $\mathrm{CV}$. 

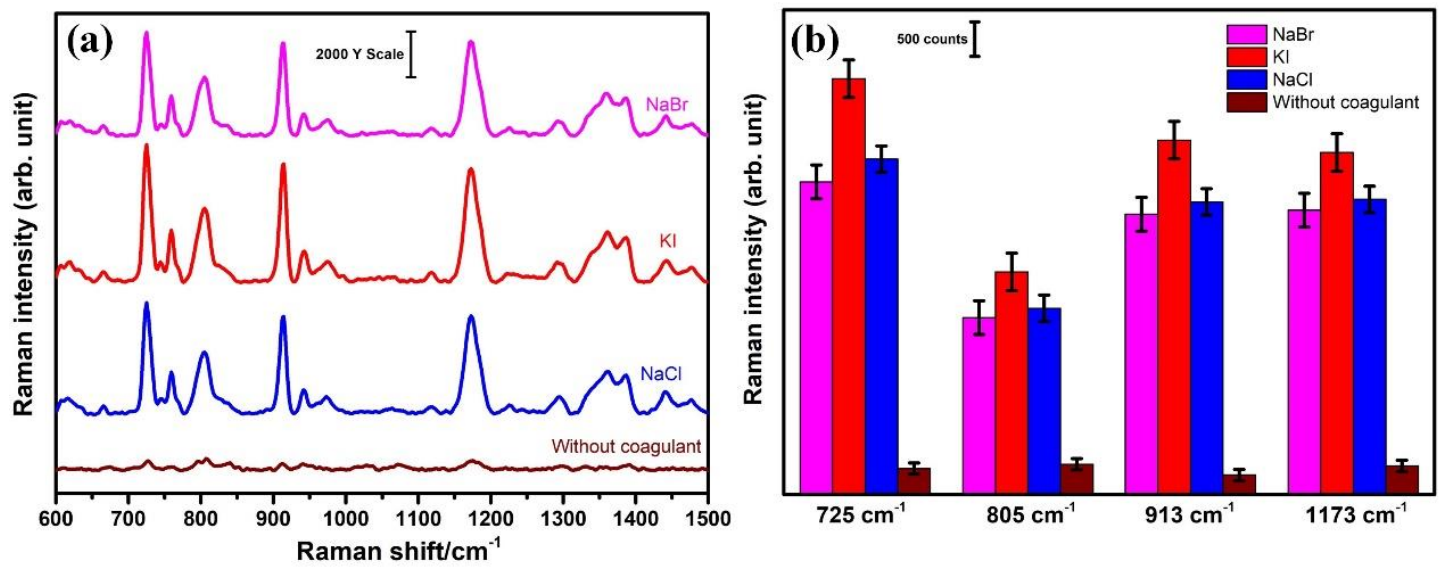

Fig. 4 (a) SERS spectrum of CV solution $\left(1 \times 10^{-6} \mathrm{M}\right)$ under the action of different aggregation agents, laser power $30 \mathrm{~mW}$, integration time $3 \mathrm{~s}$, (b) Comparison of the characteristic peak intensities of CV solution $\left(1 \times 10^{-6} \mathrm{M}\right)$ at $725,805,913$ and $1173 \mathrm{~cm}^{-1}$ under the action of different aggregation agents.
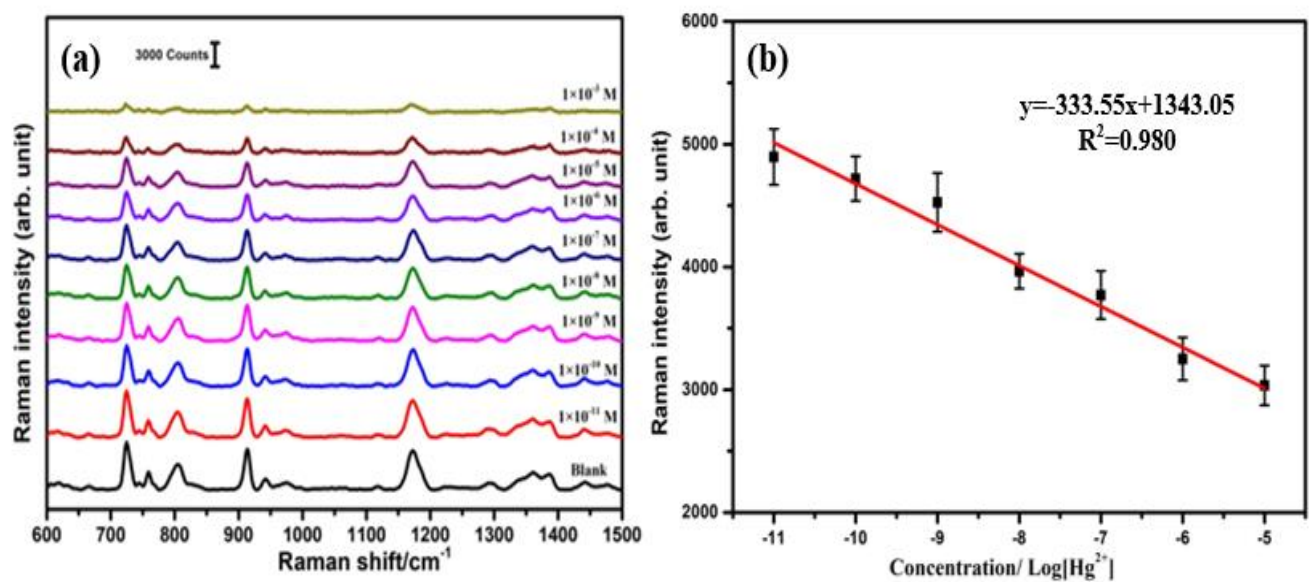

Fig. 5 (a) Using CV-AgNPs probe to detect SERS spectra of $\mathrm{Hg}^{2+}$ solutions with different concentrations, laser power $30 \mathrm{~mW}$, integration time $3 \mathrm{~s}$, (b) $\mathrm{CV}$ characteristic peak $\left(913 \mathrm{~cm}^{-1}\right)$ intensity fitted with $\mathrm{Hg}^{2+}$ concentration curve.

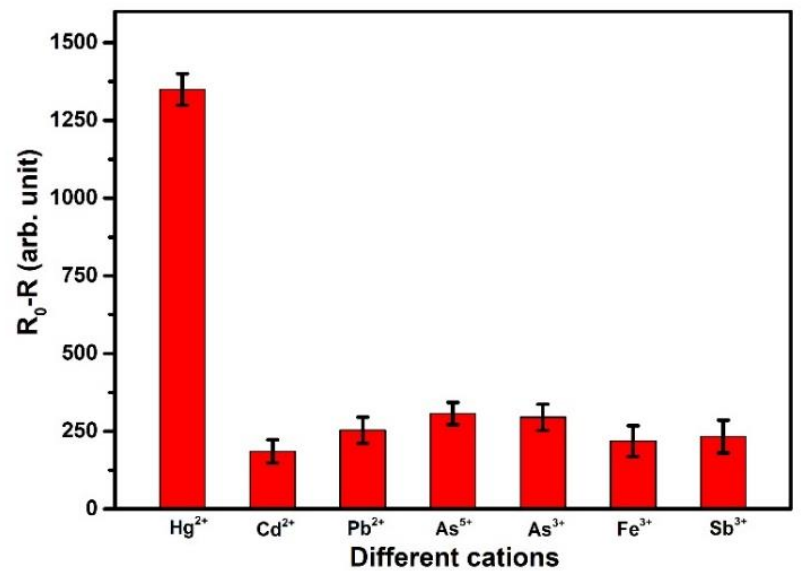

Fig. 6 Use CV-Ag NPs probe to detect different heavy metal cations, $\mathrm{R}_{0}$ and $\mathrm{R}$ are the characteristic peak intensity of CV-Ag NPs probe at $913 \mathrm{~cm}^{-1}$ before and after adding heavy metal cations, laser power $30 \mathrm{~mW}$, integration time $3 \mathrm{~s}, \mathrm{C}_{\mathrm{Hg}}{ }^{2+}: 1 \times 10^{-7} \mathrm{M}, \mathrm{C}_{\text {other cations }}: 1 \times 10^{-6} \mathrm{M}$ 


\section{Graphical Index}

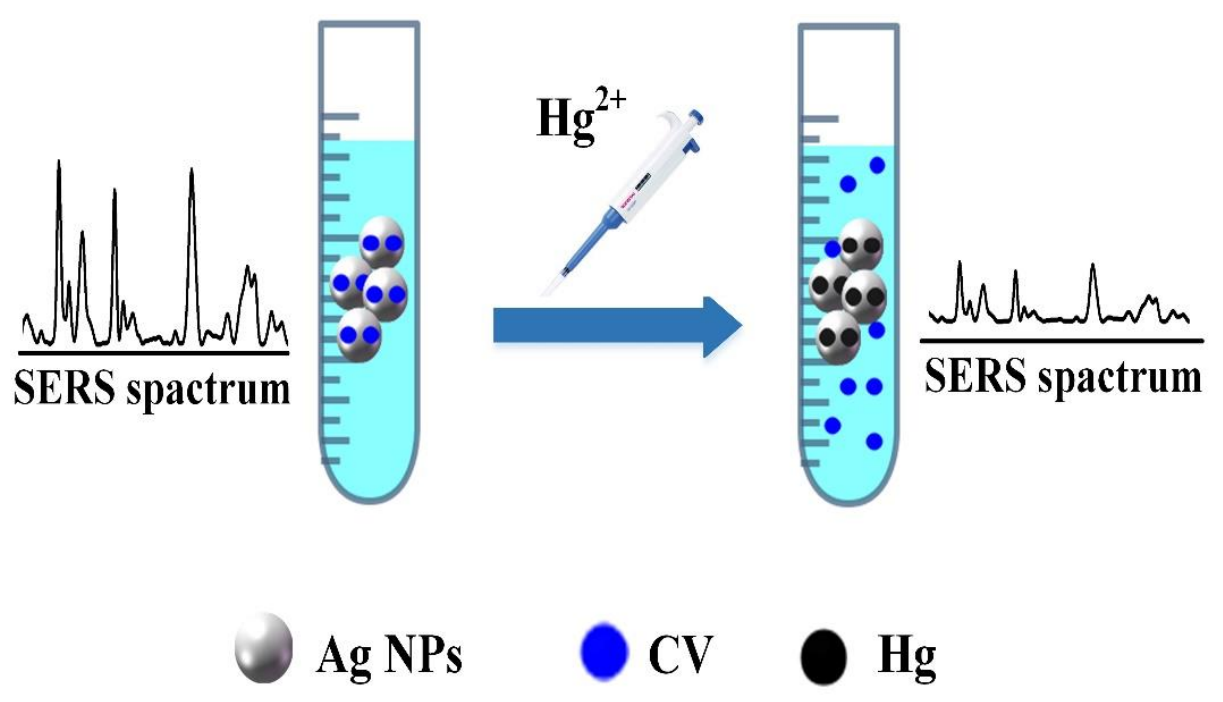

